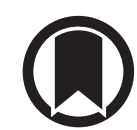

CrossMark

\section{Validation of short- and long-term demographic forecasts using the Canadian Cystic Fibrosis Registry}

\author{
To the Editor:
}

National cystic fibrosis (CF) data registries track patient characteristics over time and have provided insight into both emerging trends and current clinical needs. In a recent study, Burgel et al. [1] utilised the flow method, a demographic model that predicts future trends in populations, and forecasted a $50 \%$ increase in the Western European CF population by 2025, with the adult population experiencing the largest increase. BURGel et al. [2] subsequently used the French registry to validate short-term predictions; however, the accuracy of longer term projections has not been assessed.

The objective of this study was to assess the accuracy of short- and long-term flow method projections for the number of patients living with CF using an external data source.

This study utilised longitudinal Canadian CF Registry (CCFR) data. A detailed description of the CCFR has been published previously [3]. Ethics approval for this study was obtained from the research ethics board at St Michael's Hospital (Toronto, ON, Canada; research ethics board \# 17-119).

"Entering" and "exiting" flow rates were calculated for a particular year (t) [1]. For each year, the base population was defined as the number of patients who attended a clinic visit in that year. The "entering" flow rate was calculated as the proportion of the base population consisting of new CF diagnoses and CF patients who returned to the cohort after being lost to follow-up (defined as missing clinic visits for a 1-year period). The "exiting" flow rate was the proportion of deaths and patients lost to follow-up. To account for year-to-year variation, flow rates were averaged over a 5 -year period. To calculate the projected population for year $t+5$, the average "entering" and "exiting" flows were added to and subtracted, respectively, from the base population of year $t$.

We defined short-term forecast as 5 years in the future, whereas long-term forecast was defined as 15 years in the future. To determine the accuracy of the forecasting model, we compared the short- and long-term projections to the observed 2015 population. Short-term projections were based on the mean flow rates calculated using 2006-2010 data. Long-term projections were based on mean flow rates calculated using 1996-2000 data.

All analyses were performed using R statistical software (version 3.4.3; www.r-project.org).

The short-term projected overall number of CF patients was $4.1 \%$ higher for adults and $1.1 \%$ lower for children, in comparison to the actual registry data. Long-term estimates were less accurate, underestimating the adult population by $29.6 \%$ and the paediatric population by $7.0 \%$ (figure 1 ).

In order to understand why long-term projections were dissimilar to actual data, we examined the "entering" and "exiting" flow rates between the two time periods, namely, 1996-2000 (used to make long-term projections) and 2006-2010 (used to make short-term projections). Adult "entering" flows were similar between the two time periods; however, "exiting" flows differed. Between 1996-2000 and 20062010 , the mean death rate decreased from $3.6 \%$ to $2.1 \%$. The mean loss to follow-up rate also decreased from $6.0 \%$ (1996-2000) to 4.7\% (2006-2010). The paediatric new CF diagnosis rate increased slightly from

@ERSpublications

Based on the results of this study, a model that takes into consideration changing rates over time is needed to accurately estimate future populations http://bit.ly/35IDhQ0

Cite this article as: Martelli V, Sykes J, Burgel P-R, et al. Validation of short- and long-term demographic forecasts using the Canadian Cystic Fibrosis Registry. Eur Respir J 2020; 55: 1901667 [https://doi.org/ 10.1183/13993003.01667-2019]. 

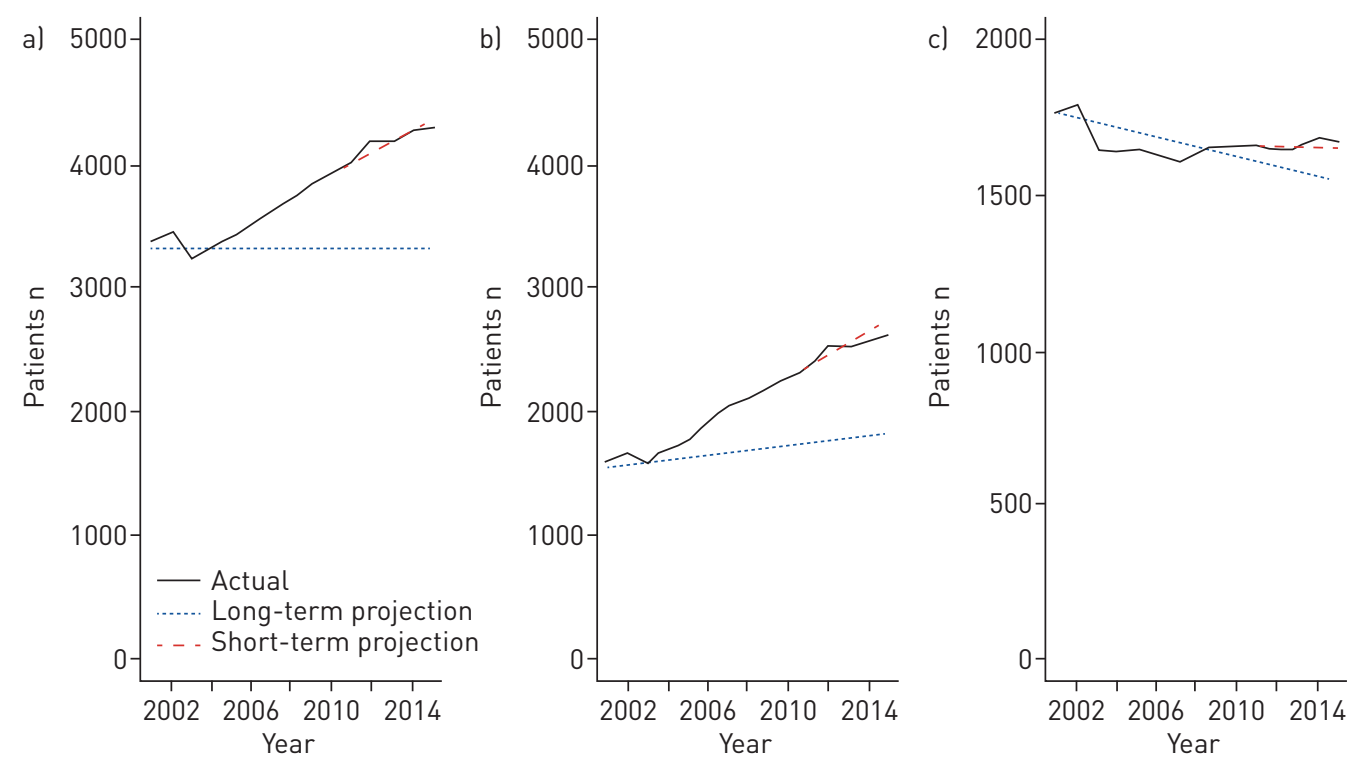

FIGURE 1 Actual number versus projections (short- and long-term) of number of cystic fibrosis patients. a) Overall estimates; b) adult estimates; c) paediatric estimates.

$5.8 \%$ in $1996-2000$ to $7.2 \%$ in $2006-2010$. The paediatric death rate remained relatively stable over the study period (0.5\% in 1996-2000 and $0.4 \%$ in 2006-2010).

Using the flow method, short-term projections of the number of $\mathrm{CF}$ patients were within $5 \%$ of the actual values. However, when forecasting out 15 years, the methodology was inaccurate by as much as $30 \%$.

All forecasting methods have limitations as they are predicting the unknown. The flow method assumes that entering and exiting flow rates remain constant over time. When this method was applied to Canadian CF population data, this assumption held true over a short time period; however, this was not the case over longer durations. In a population in which survival rates are continually improving, the long-term projections will be underestimated. Deaths due to CF are uncommon in the paediatric population. Therefore, improved survival will result in growth of the adult population preferentially. Underestimation of the future demographics of the CF population could result in insufficient infrastructure and resources to care adequately for adults living with this disease. Future resource planning should specifically target adults living with this disease. In previously published work, Burgel et al. [1] initially validated the flow method by calculating a 2-year forecast and comparing the results to 2012 data from the French CF Registry, demonstrating a small (0.5\%) overestimation. In a subsequent publication, a 6-year forecast was calculated using mean flow rates from 2003 to 2009, and forecasted results were compared to the actual 2015 French CF Registry data, with a 1.9\% underestimation [2]. Based on our results, long-term projections using historical data will be inaccurate if flow rates change substantially over time, as this methodology does not account for changes in demographic trends.

In conclusion, the flow method assumes that flow rates will remain constant [1]. Over the studied time period, using Canadian registry data, flow rates remained relatively constant over 5 years; however, this was not the case over 15 years. CF care is rapidly evolving and new therapies are being developed, such as CFTR modulators. An observational study by Bessonova et al. [4] reported that ivacaftor-treated patients have a decreased risk of death compared to comparator-matched patients, suggesting that these treatments will further improve health outcomes and result in further changes to the demographics of the future CF population. As such, a dynamic long-term forecasting method that takes into consideration changing rates over time is critical to enable accurate predictions, which will allow for resource planning to meet the needs of the changing CF population [5].

Vanessa Martelli $\circledast^{1,2}$, Jenna Sykes ${ }^{2}$, Pierre-Régis Burgel $\oplus^{3,4,5,6}$, Gil Bellis ${ }^{4,7}$, Adèle Coriati ${ }^{2}$, Sanja Stanojevic ${ }^{8}$ and Anne Louise Stephenson ${ }^{1,2}$

${ }^{1}$ Dept of Medicine, University of Toronto, Toronto, ON, Canada. ${ }^{2}$ Adult Cystic Fibrosis Program, Division of Respirology, St Michael's Hospital, Toronto, ON, Canada. ${ }^{3}$ Cochin Hospital, Assistance Publique-Hôpitaux de Paris, Paris, France. ${ }^{4}$ Inserm U1016 Institut Cochin, Paris, France. ${ }^{5}$ Université de Paris, Paris, France. ${ }^{6}$ ERN Lung CF Network. ${ }^{7}$ Institut National d'Études Démographiques, Paris, France. ${ }^{8}$ Division of Respiratory Medicine, The Hospital for Sick Children, Toronto, ON, Canada. 
Correspondence: Anne L. Stephenson, St Michael's Hospital, 30 Bond Street, 6th Floor, Bond Wing, Toronto, ON, M5B 1W8, Canada. E-mail: anne.stephenson@unityhealth.to

Received: 21 Aug 2019| Accepted after revision: 10 Oct 2019

Acknowledgements: We thank Cystic Fibrosis Canada for providing access to Canadian CF Registry data for this study, and we thank individuals living with CF and their families for allowing their data to be collected in the CF Registry to be used for clinical research. This work was presented as a poster presented at the North American Cystic Fibrosis Conference, Denver, Colorado, 18-21 October 2018.

Support statement: This work was supported by Cystic Fibrosis Canada (grant 599819) and Vaincre la Mucoviscidose. Funding information for this article has been deposited with the Crossref Funder Registry.

Conflict of interest: V. Martelli has nothing to disclose. J. Sykes has nothing to disclose. P-R. Burgel reports grants and personal fees from Boehringer Ingelheim, personal fees from AstraZenaca, Chiesi, GSK, Novartis, Pfizer, Teva, Vertex and Zambon, outside the submitted work. G. Bellis has nothing to disclose. A. Coriati has nothing to disclose. S. Stanojevic has nothing to disclose. A.L. Stephenson reports grants and personal fees from Cystic Fibrosis Canada, personal fees from Vertex Pharmceuticals, outside the submitted work.

\section{References}

1 Burgel PR, Bellis G, Olesen HV, et al. Future trends in cystic fibrosis demography in 34 European countries. Eur Respir J 2015; 46: 133-141.

2 Burgel PR, Bellis G, Elborn JS. Modelling future trends in cystic fibrosis demography using the French Cystic Fibrosis Registry: update and sensitivity analysis. Eur Respir J 2017; 50: 1700763.

3 Stephenson AL, Sykes J, Stanojevic S, et al. Survival comparison of patients with cystic fibrosis in Canada and the United States: a population-based cohort study. Ann Intern Med 2017; 166: 537-546.

4 Bessonova L, Volkova N, Higgins M, et al. Data from the US and UK cystic fibrosis registries support disease modification by CFTR modulation with ivacaftor. Thorax 2018; 73: 731-740.

5 Meira-Machado L, de Uña-Alvarez J, Cadarso-Suárez C, et al. Multi-state models for the analysis of time-to-event data. Stat Methods Med Res 2009; 18: 195-222. 